\title{
Atuação multiprofissional em face ao cuidado à criança hospitalizada: uma revisão integrativa
}

\author{
Multiprofessional team action in the face of care for hospitalized children: an integrative review \\ Acción multiprofesional ante la atención al niño hospitalizado: una revisión integradora
}

Recebido: 17/02/2021 | Revisado: 23/02/2021 | Aceito: 24/02/2021 | Publicado: 06/03/2021

Richard Morrinson Couras de Carvalho

ORCID: https://orcid.org/0000-0002-2564-3657 Residência Multiprofissional em Saúde da Criança, Brasil E-mail: rich_couras@hotmail.com

Itacely Marinho da Silva

ORCID: https://orcid.org/0000-0002-0851-1687 Residência Multiprofissional em Saúde da Criança, Brasil E-mail: itacelymarinho@hotmail.com

Emanuely Rayane dos Santos Martins

ORCID: https://orcid.org/0000-0002-8888-2188 Residência Multiprofissional em Saúde da Criança, Brasil E-mail: emanuelyray@gmail.com

Cibério Macedo Landim

ORCID: https://orcid.org/0000-0002-0824-4056

Residência Multiprofissional em Saúde da Criança, Brasil E-mail: ciberiolandim@hotmail.com

Priscilla Alves Nobrega Gambarra

ORCID: https://orcid.org/0000-0003-3327-377X

Residência Multiprofissional em Saúde da Criança, Brasil E-mail: priscilla_ang@hotmail.com

Josemery Amaro de Melo

ORCID: https://orcid.org/0000-0001-5884-0545 Residência Multiprofissional em Saúde da Criança, Brasil E-mail: jhosimery@gmail.com

Rossana Gois Ferreira

ORCID: https://orcid.org/0000-0001-8541-7929

Residência Multiprofissional em Saúde da Criança, Brasil

E-mail: rossanagoisf@gmail.com

\begin{abstract}
Resumo
O objetivo deste estudo foi investigar a atuação da equipe multiprofissional. Foi realizada uma revisão integrativa da literatura, tendo como base a busca de artigos científicos indexados as bases de dados Pubmed, ScienceDirect, Lilacs e Scielo, utilizando como descritores: equipe multiprofissional, hospitalização e criança, combinados com operadores booleanos, bem como suas traduções para o inglês e espanhol. Realizada a busca foram encontradas 4.295 produções científicas, aplicando filtros e critérios estabelecidos de inclusão e exclusão o resultado final foi de 19 artigos que compuseram amostra. A partir dos achados, a discussão foi categorizada em tópicos que envolvem a atuação das práticas multiprofissionais, tais quais: equipe Multiprofissional; Relações interpessoais: equipe multiprofissional e família; Desafios e possibilidade das práticas multiprofissionais. O processo de trabalho da equipe multiprofissional está relacionado diretamente à concepção dos profissionais acerca do significado do trabalho em equipe e da importância no desenvolvimento e qualidade da assistência prestada. Para o êxito é necessário atenção direcionada às demandas que emergem no cotidiano hospitalar, assim como, propiciar o fortalecimento dos vínculos equipe-equipe, como também, equipe-família, desse modo, possibilitar comunicação eficaz proporcionando à criança hospitalizada um cuidado integral e assertivo a partir da associação destas práticas.
\end{abstract}

Palavras-chave: Equipe multiprofissional; Hospitalização e criança.

\begin{abstract}
The aim of this study was to investigate the performance of the multiprofessional team; Interpersonal relationships: multiprofessional team and family and the challenges and possibilities of multiprofessional practices. A systematic review of the literature was carried out, based on the search for scientific articles indexed in the databases Pubmed, ScienceDirect, Lilacs and Scielo, using as descriptors: multiprofessional team, hospitalization and child, combined with Boolean operators, as well as their translations for the English and Spanish. After the search, 4,295 scientific productions were found, applying filters and establishing the inclusion and exclusion criteria, the final result was 19
\end{abstract}


articles that comprised the sample. Based on the findings, the discussion was categorized into themes that involve the performance of multiprofessional practice, such as: Multiprofessional team; Interpersonal relationships: multiprofessional team and family; Challenges and the possibility of multiprofessional practices. The work process of the multidisciplinary team is directly related to the professionals' conception of the meaning of teamwork and the importance in the development and quality of the assistance provided. For success, it is necessary to focus attention on the demands that emerge in the hospital routine, as well as providing the strengthening of team-team bonds, as well as team-family, thus enabling effective communication providing the hospitalized child with comprehensive and assertive care from the association of these practices.

Keywords: Multiprofessional team; Hospitalization and child.

\section{Resumen}

El objetivo de este estudio fue investigar el desempeño del equipo multiprofesional; Relaciones interpersonales: equipo multiprofesional y familia y los desafíos y posibilidad de prácticas multiprofesionales. Se realizó una revisión bibliográfica sistemática, a partir de la búsqueda de artículos científicos indexados a las bases de datos Pubmed, ScienceDirect, Lilacs y Scielo, utilizando como descriptores: equipo multiprofesional, hospitalización y niño, combinado con operadores booleanos, así como sus traducciones al inglés. y español. Luego de la búsqueda, se encontraron 4.295 producciones científicas, aplicando filtros y criterios de inclusión y exclusión establecidos, el resultado final fue de 19 artículos que conformaron la muestra. Con base en los hallazgos, la discusión se categorizó en temas que involucran el desempeño de prácticas multiprofesionales, tales como: Equipo multiprofesional; Relaciones interpersonales: equipo multiprofesional y familia; Retos y posibilidad de prácticas multiprofesionales. El proceso de trabajo del equipo multiprofesional está directamente relacionado con la concepción de los profesionales sobre el significado del trabajo en equipo y la importancia en el desarrollo y calidad de la asistencia brindada. Para el éxito es necesario centrar la atención en las demandas que surgen en la rutina hospitalaria, además de brindar el fortalecimiento de los lazos equipo-equipo, así como equipo-familia, posibilitando así una comunicación efectiva brindando al niño hospitalizado una atención integral y asertiva a de la asociación de estas prácticas.

Palabras clave: Equipo multiprofesional; Hospitalización y infancia.

\section{Introdução}

O SUS em sua essência tem como princípios direcionar aos usuários um serviço com equidade, integralidade e universalidade (Brasil, 1990), os quais norteiam a prática profissional de todos os agentes envolvidos a fim de que sejam cumpridas de forma resolutiva e humanizadas. Todavia, a aplicação prática desses princípios traz desafios importantes, como a condução de um desempenho interprofissional qualificado que proporcione educação mútua, a partir de discussão direcionada a uma conduta conjunta, levando em consideração a competência de cada área profissional, visando um mesmo objetivo. Contudo, a formação base de profissionais de saúde no Brasil tende a proporcionar uma fragmentação de conhecimento e consequentemente individualização do cuidado (Arnemann et al., 2018).

A integralidade, conforme a Lei 8080 de 19 de setembro de 1990 compreende "um conjunto de ações e serviços preventivos e curativos, individuais e coletivos, exigidos para cada caso em todos os níveis de complexidade do sistema", que em sua variedade de vertentes, aponta atributos e valores a respeito de particularidades que são almejadas dentro das práticas de saúde. Desse modo, torna-se uma importante motivação e principal justificativa para sugestionar o trabalho em equipe frente ao trabalho individualizado.

Aos profissionais envolvidos na equipe de saúde é preciso e necessário delinear uma alternativa de inserção e de organização de trabalho dentro do serviço, que substitua e supere o modelo biomédico hegemônico de atuação para que possibilite uma articulação de ideias que propiciem a promoção, prevenção e recuperação nos diversos níveis de complexidade que envolvam as necessidades de saúde dos sujeitos (Peduzzi et al., 2007).

Nesse sentido, a equipe multiprofissional imprime uma proposta de trabalho que incorpora os diferentes saberes profissionais, desempenhando uma atuação articulada e integrada. Desse modo, para o desenvolvimento das práticas multiprofissionais é imprescindível a receptividade e compreensão do conhecimento que envolve as profissões que compõem a equipe, com o objetivo de garantir a homogeneidade de ações e reflexões proporcionando uma intervenção fecunda (Miareli, 2012). 
Assim, é atribuído a essa equipe identificar demandas e apreendê-las de modo que ultrapassem os aspectos individuais e possam ser observadas a partir da totalidade que o sujeito está imerso, a começar na percepção dos fatores que implicam diretamente no processo de adoecimento. Em consequência a isso, possibilite a oferta de uma atenção resolutiva ao processo de saúde-doença (Arnemann et al., 2018).

No contexto pediátrico, é imprescindível a atuação de equipe multiprofissional, uma vez que o processo de hospitalização infantil envolve muitos aspectos, tais quais: físicos, sociais, culturais e emocionais, potencializados pelo ambiente hospitalar que gera medo e remete à dor. Dessa maneira, os profissionais envolvidos no cuidado da criança se deparam com uma demanda extensa e delicada exigindo uma compreensão de todo o contexto que a envolve (Souza, et.al; 2016).

Expostas essas razões e diante da importância do protagonismo dessas equipes mediante o cuidado, essa pesquisa se desenvolve com o objetivo de apresentar a atuação multiprofissional frente à criança hospitalizada, bem como a importância da família neste contexto, além da possibilidade de práticas multiprofissionais visando à qualidade de vida destes pacientes.

\section{Metodologia}

Trata-se de uma pesquisa qualitativa do tipo revisão integrativa da literatura, tipo de pesquisa que possibilita a síntese dos estudos publicados sobre determinado tema, obtendo, a partir deles, conclusões que irão contribuir com as diversas tomadas de decisão (Pereira, Shitsuka, Parreira \& Shitsuka, 2018; Carvalho, Ferreira \& Ferreira, 2020). Foi elaborada conforme as instruções contidas no Preferred Reporting Items for Systematic reviews and Meta-Analyses (PRISMA), a qual utiliza uma lista de verificação aplicada no decorrer da revisão, com o intuito de aprimorar os relatos obtidos (Liberati et al., 2009).

Para a operacionalização da presente revisão foram consideradas seis etapas: identificação do tema e seleção da questão norteadora; estabelecimento dos critérios de inclusão e exclusão; seleção dos artigos; categorização dos artigos; análise dos estudos; e síntese do conhecimento. (Carvalho, Ferreira \& Ferreira, 2020).

Para guiar a revisão integrativa, formulou-se a seguinte questão norteadora: "Como ocorre à atuação multiprofissional frente ao cuidado da criança hospitalizada?". A seleção da amostra deu-se por meio do acesso as seguintes bases de dados: Biblioteca Nacional de Medicina (PubMed), ScienceDirect, Ciências Sociais e da Saúde da América Latina e do Caribe (LILACS) e Biblioteca Eletrônica Científica Online (SciELO). Utilizou-se como estratégia de busca os descritores controlados ("Equipe Multiprofissional", "Hospitalização" e "Criança"), combinados com operadores booleanos, (("Patient Care Team") AND (Hospitalization) AND (Child)), dispostos no Descritores em Ciências da Saúde (DesC) e Medical SubjectHeadings (MeSH).

Em cada etapa de elaboração da pesquisa, tanto a busca, a seleção, a extração, quanto análise dos dados foram realizadas aos pares, seguida de discussão entre os autores, que elegeram as publicações mais pertinentes para integrarem o estudo. No caso de qualquer discordância, os autores entraram em um consenso para resolução da possível divergência.

Como critérios de inclusão, utilizaram-se os artigos na categoria original, disponibilizados na íntegra, publicados nos idiomas português, inglês e espanhol, e que envolva o público pediátrico, com intervalo de 2010 a 2020. Por sua vez, foram critérios de exclusão: Artigos que não atendessem ao objetivo do estudo; publicados nos formatos de trabalho de conclusão de curso, resenha e resumo de congresso, relato de caso, revisões da literatura; e que estivessem repetidos nas bases de dados. O processo de seleção dos artigos, bem como os resultados, está apresentado na Figura 1. 
Figura 1: Fluxograma Explicativo Da Estratégia De Busca E Seleção Dos Estudos, João Pessoa, 2020.

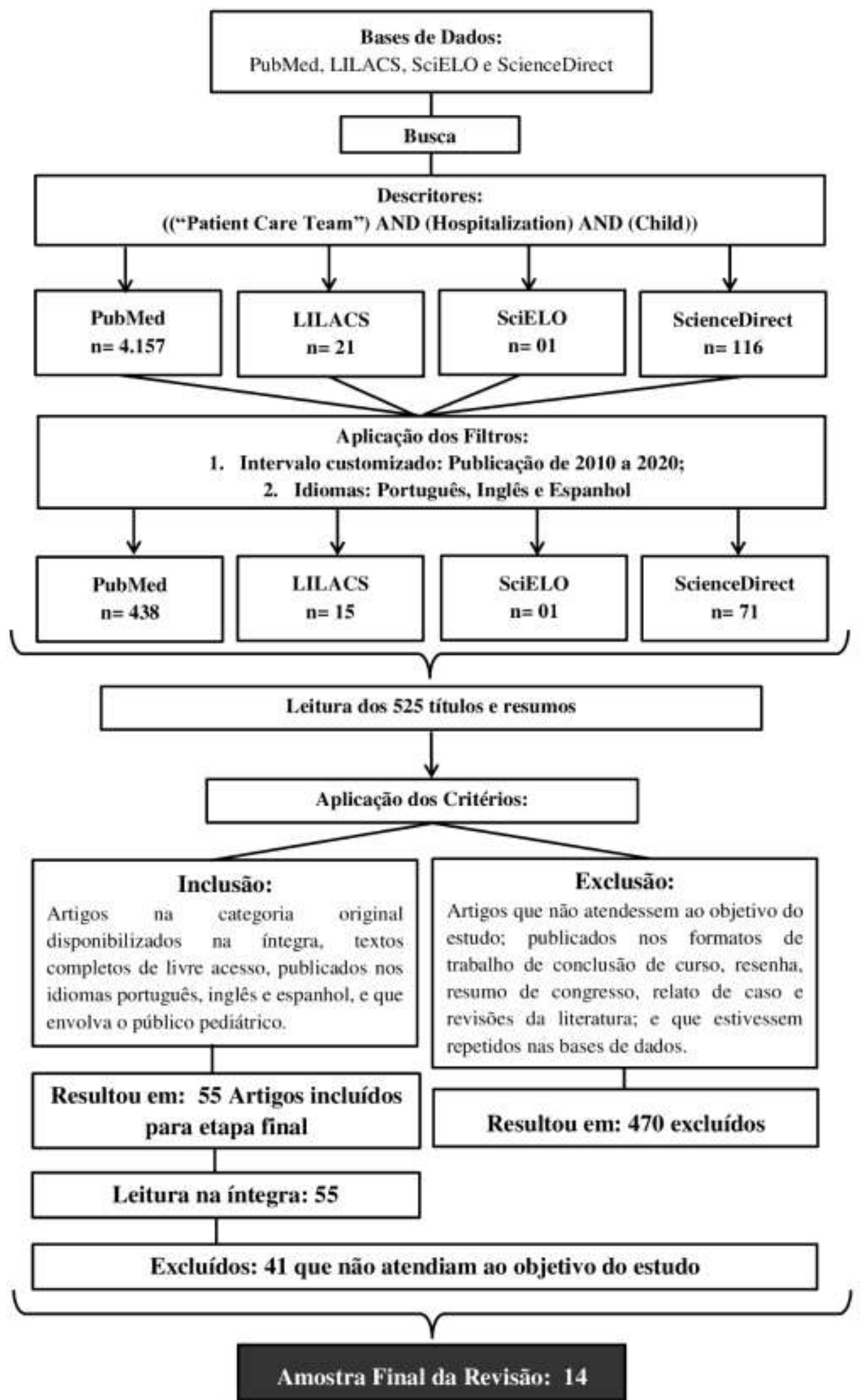

Fonte: Autores (2020). 


\section{Resultados}

Ao utilizar os descritores combinados entre si nas buscas nos bancos de dados, foram encontradas 4.295 produções científicas. Ao aplicar filtro de tempo de publicação e idioma, restaram 525 artigos. Em seguida, foi realizada a leitura dos títulos e resumos com aplicação dos critérios de inclusão e exclusão, onde restaram 55 artigos para leitura na íntegra. Após leitura cuidadosa e criteriosa desta produção, 14 artigos cumpriram os critérios estabelecidos. Os resultados da produção científica que compôs a amostra final, com título, autores, país, objetivos e resultados, estão descritos no Quadro 1 seguinte.

Quadro 1: Distribuição Dos Artigos Selecionados, João Pessoa, 2020.

\begin{tabular}{|c|c|c|}
\hline Título, autor e país & Objetivos do estudo & Resultados \\
\hline $\begin{array}{c}\text { Doença crônica } \\
\text { infantojuvenil: vínculo } \\
\text { profissional-família para a } \\
\text { promoção do apoio social } \\
\text { Machado, N. A. et al. } \text { (2018) } \\
\text { Brasil }\end{array}$ & $\begin{array}{c}\text { Analisar o vínculo na promoção do } \\
\text { apoio social ofertado pela equipe } \\
\text { multiprofissional de saúde às famílias } \\
\text { durante a hospitalização de } \\
\text { crianças/adolescentes com doença } \\
\text { crônica }\end{array}$ & $\begin{array}{l}\text { Evidenciaram-se dificuldades como a } \\
\text { fragilidade na comunicação e interação entre } \\
\text { equipe-família; lacunas na organização do } \\
\text { processo de trabalho e falta de infraestrutura } \\
\text { hospitalar, que interferem na oferta de apoio }\end{array}$ \\
\hline $\begin{array}{c}\text { Desenvolvimento de um } \\
\text { Programa Multidisciplinar de } \\
\text { Vias Aéreas Pediátricas: Uma } \\
\text { Experiência Institucional. } \\
\text { Dalesio, et al. (2019) } \\
\text { Brasil }\end{array}$ & $\begin{array}{c}\text { Desenvolver um programa pediátrico } \\
\text { de vias aéreas difíceis (PDART) para } \\
\text { otimizar o manejo seguro de } \\
\text { emergências de vias aéreas em } \\
\text { crianças }\end{array}$ & $\begin{array}{l}\text { Com a implementação do PDART houve a } \\
\text { redução de } 51 \text { para } 19 \text { emergências } \\
\text { pediátricas de vias aéreas, bem como menos } \\
\text { chamadas de emergências PDART em média } \\
\text { ( } 11 \text { por ano antes do PDART em } \\
\text { comparação com } 7 \text { por ano após o início do } \\
\text { programa) }\end{array}$ \\
\hline $\begin{array}{l}\text { Interprofessional Teams: } \\
\text { Current Trends and Future } \\
\text { Directions } \\
\text { Baird, J.; Ashland M.; } \\
\text { Rosenbluth, G. (2019) } \\
\text { Estados Unidos }\end{array}$ & $\begin{array}{l}\text { Identificar as vantagens e desafios de } \\
\text { se trabalhar em um ambiente } \\
\text { interprofissional, os principais } \\
\text { componentes do trabalho em equipe } \\
\text { interprofissional e alguns exemplos } \\
\text { de modelos bem-sucedidos }\end{array}$ & $\begin{array}{l}\text { As equipes interprofissionais ajudam a } \\
\text { diminuir as práticas clínicas e operacionais } \\
\text { tradicionalmente isoladas dentro de } \\
\text { organizações de saúde e pode ajudar no } \\
\text { achatamento do poder hierárquico estrutural. } \\
\text { A prática interprofissional requer uma } \\
\text { mudança de estruturas de trabalho paralelas } \\
\text { para engajamento colaborativo e sinérgico, a } \\
\text { realização das mesmas, requerem um } \\
\text { investimento na educação interprofissional } \\
\text { para estagiários e clínicos em atividade. As } \\
\text { organizações devem considerar as } \\
\text { oportunidades de envolvimento da família } \\
\text { tanto na clínica quanto equipes operacionais }\end{array}$ \\
\hline $\begin{array}{l}\text { Challenges in conducting } \\
\text { multicentre, multidisciplinary, } \\
\text { longitudinal studies in } \\
\text { children with chronic } \\
\text { conditions } \\
\text { Broder, H. L. et al. }(2017) \\
\text { Estados Unidos }\end{array}$ & $\begin{array}{l}\text { Identificar os desafios encontrados e } \\
\text { as soluções buscadas em um estudo } \\
\text { longitudinal observacional recém- } \\
\text { concluído de crianças com fissura } \\
\text { labiopalatina, bem como seus } \\
\text { cuidadores }\end{array}$ & $\begin{array}{c}\text { A comunicação contínua dentro e entre os } \\
\text { locais e o comprometimento e a flexibilidade } \\
\text { do investigador e do clínico foram } \\
\text { estratégias eficazes para atingir os objetivos } \\
\text { propostos }\end{array}$ \\
\hline $\begin{array}{l}\text { Chronic non-cancer pain in } \\
\text { children: we have a problem, }\end{array}$ & $\begin{array}{l}\text { Avaliar as condições associadas e a } \\
\text { evolução da doença crônica } \\
\text { dor, juntamente com o papel crucial de } \\
\text { cada membro de uma clínica }\end{array}$ & $\begin{array}{l}\text { A partir da abordagem psicossocial, por } \\
\text { meio da equipe multiprofissional, o estudo } \\
\text { apontou uma melhoria do funcionamento em }\end{array}$ \\
\hline
\end{tabular}




\begin{tabular}{|c|c|c|}
\hline $\begin{array}{l}\text { but also solutions } \\
\text { Vega, et al. } \\
\text { (2018) } \\
\text { Colombia }\end{array}$ & $\begin{array}{l}\text { multidisciplinar de dor crônica } \\
\text { envolvida no cuidado do } \\
\text { crianças e adolescentes com dor } \\
\text { crônica não oncológica }\end{array}$ & crianças e adolescentes com dor crônica \\
\hline $\begin{array}{l}\text { Distributed expertise: } \\
\text { qualitative study of a British } \\
\text { network of multidisciplinary } \\
\text { teams supporting parents of } \\
\text { children with chronic kidney } \\
\text { disease. } \\
\text { Swallow, V. et al. (2014) } \\
\text { Grã-Bretanha }\end{array}$ & $\begin{array}{l}\text { Relatar um aspecto de um estudo das } \\
\text { interações entre profissionais e pais } \\
\text { em uma rede de } 12 \text { unidades renais } \\
\text { infantis na Grã-Bretanha }\end{array}$ & $\begin{array}{l}\text { Observou-se que quatro temas emergiram } \\
\text { relacionados à forma como a expertise foi } \\
\text { distribuída dentro e entre as equipes } \\
\text { multidisciplinares (MDTs): (i) } \\
\text { reconhecimento da expertise de cada um, (ii) } \\
\text { compartilhamento de expertise dentro das } \\
\text { MDTs, (iii) interpretação da linguagem e (iv) } \\
\text { atuação como corretores }\end{array}$ \\
\hline $\begin{array}{l}\text { Falla intestinal en el paciente } \\
\text { pediátrico: experiencia y } \\
\text { manejo por un grupo } \\
\text { multidisciplinario } \\
\text { Giraldo, et al. }(2015) \\
\text { Colombia }\end{array}$ & $\begin{array}{l}\text { Caracterizar o manejo multidisciplinar } \\
\text { e os resultados em pacientes } \\
\text { pediátricos com insuficiência intestinal }\end{array}$ & $\begin{array}{c}\text { A autonomia intestinal foi alcançada em } \\
69,7 \% \text { dos casos, } 72,7 \% \text { deles apresentaram } \\
\text { melhora no escore } \mathrm{z} \text { de peso e apresentavam } \\
\text { albumina final significamente superior ao } \\
\text { valor inicial }\end{array}$ \\
\hline $\begin{array}{l}\text { Interprofessional Teamwork } \\
\text { During Family Meetings in } \\
\text { the Pediatric Cardiac Intensive } \\
\text { Care Unit } \\
\text { Walter, et.al (2019) } \\
\text { Estados Unidos }\end{array}$ & $\begin{array}{c}\text { Avaliar quantitativa e qualitativamente } \\
\text { as contribuições feitas por membros da } \\
\text { equipe de diferentes profissões na } \\
\text { comunicação com os pais durante as } \\
\text { reuniões de família }\end{array}$ & $\begin{array}{l}\text { Em } 10 \text { reuniões, os médicos falaram em } \\
\text { média } 78,1 \% \text { (DP 10,7\%) de cada reunião, } \\
\text { os não médicos 9,6\% (DP 7,8\%) e os pais } \\
17,4 \% \text { (DP } 12,2 \% \text { ). A compreensão dos pais } \\
\text { foi avaliada em média } 0,2 \text { (DP } 0,4 \text { ) vezes por } \\
\text { reunião. Os pais expressaram emoção em } \\
\text { média } 4,2 \text { vezes por reunião (DP } 7,1 \text { ), e a } \\
\text { equipe clínica respondeu empaticamente } 2,2 \\
\text { vezes por reunião (DP } 4,3 \text { ). Todas as } \\
\text { respostas empáticas do clínico foram uma } \\
\text { minoria de suas contribuições gerais. A } \\
\text { conversa era quase exclusivamente entre } \\
\text { médicos e familiares, até que os médicos } \\
\text { indicaram que outros membros da equipe } \\
\text { poderiam contribuir }\end{array}$ \\
\hline $\begin{array}{c}\text { Abordagem Multidisciplinar } \\
\text { Para Pacientes Com Cistinose } \\
\text { Neforpática: Modelo Para } \\
\text { Atendiemnto Em Uma Doença } \\
\text { Renal Rara E Crônica } \\
\text { Vaisbich, et al. }(2018) \\
\text { Brasil }\end{array}$ & $\begin{array}{l}\text { Alcançar o maior número de } \\
\text { profissionais envolvidos no } \\
\text { seguimento de pacientes pediátricos, } \\
\text { fortalecendo as bases para a criação de } \\
\text { um protocolo nacional, observando-se } \\
\text { as particularidades de cada caso. }\end{array}$ & $\begin{array}{l}\text { Apresentação do protocolo de seguimento, o } \\
\text { qual vem sendo empregado com melhora na } \\
\text { qualidade da assistência e consistem de uma } \\
\text { abordagem multidisciplinar, incluindo, } \\
\text { principalmente, atendimento prestado por } \\
\text { médico, enfermeiro, psicólogo, nutricionista } \\
\text { e assistente social. }\end{array}$ \\
\hline $\begin{array}{l}\text { Cuidados paliativos em } \\
\text { oncologia pediátrica: } \\
\text { percepções, saberes e prática } \\
\text { na perspectiva da equipe } \\
\text { multiprofissional } \\
\text { Silva, et al. } \\
\text { (2015) }\end{array}$ & $\begin{array}{l}\text { Conhecer as percepções, saberes e } \\
\text { práticas da equipe multiprofissional na } \\
\text { atenção às crianças em cuidados } \\
\text { paliativos em unidade de oncologia } \\
\text { pediátrica }\end{array}$ & $\begin{array}{l}\text { Da análise emergiram quatro temas } \\
\text { intitulados cuidados paliativos: concepções } \\
\text { da equipe multiprofissional; a construção de } \\
\text { um cuidado singular; as facilidades e as } \\
\text { dificuldades vivenciadas pela equipe e } \\
\text { aprendizagens significativas }\end{array}$ \\
\hline
\end{tabular}




\begin{tabular}{|c|c|c|}
\hline Brasil & & \\
\hline $\begin{array}{l}\text { Characteristics of children and } \\
\text { adolescents at the } \\
\text { Switzerland-wide first } \\
\text { ambulatory interdisciplinary } \\
\text { pain clinic at the University } \\
\text { Children's Hospital Basel - a } \\
\text { retrospective study } \\
\text { Schneider, T et al. (2019) } \\
\text { Suíça }\end{array}$ & $\begin{array}{l}\text { Avaliar as características dos pacientes } \\
\text { do primeiro ambulatório } \\
\text { interdisciplinar para crianças e } \\
\text { adolescentes com dor crônica na Suíça } \\
\text { e compará-los com dados de outros } \\
\text { centros internacionais }\end{array}$ & $\begin{array}{l}\text { Verificou-se que a média de tempo antes do } \\
\text { encaminhamento para tratamento paciente } \\
\text { infanto-juvenil com síndromes dolorosas } \\
\text { complexas era alta (cerca de } 2 \text { anos). Os } \\
\text { pacientes com cefaleia estavam sub- } \\
\text { representados no Centro de Basel comparado } \\
\text { a outros centros }\end{array}$ \\
\hline $\begin{array}{l}\text { Grupo de apoio aos pais como } \\
\text { uma experiência } \\
\text { transformadora para a família } \\
\text { em unidade neonatal } \\
\text { Balbino, F. S. et al. }(2015) \\
\text { Brasil }\end{array}$ & $\begin{array}{l}\text { Compreender o significado da } \\
\text { participação da família no Grupo de } \\
\text { Apoio aos Pais na unidade neonatal }\end{array}$ & $\begin{array}{l}\text { Foi evidenciado que o "grupo de pais" é } \\
\text { uma experiência transformadora para a } \\
\text { família, pois promove o esclarecimento de } \\
\text { suas dúvidas, representa apoio durante a } \\
\text { hospitalização do recém-nascido, favorece a } \\
\text { troca de experiência entre os pais e maior } \\
\text { interação entre a equipe de saúde e a família }\end{array}$ \\
\hline $\begin{array}{l}\text { Minding the gap: } \\
\text { Interprofessional } \\
\text { communication during } \\
\text { inpatient and post discharge } \\
\text { chasm care } \\
\text { Scotten, M } \text { et al. }(2015) \\
\text { Estados Unidos }\end{array}$ & $\begin{array}{l}\text { Mostrar a importância de uma boa } \\
\text { comunicação entre a equipe } \\
\text { multiprofissional e os familiares } \\
\text { mesmo após alta hospitalar }\end{array}$ & $\begin{array}{l}\text { O nível de satisfação dos pais e pacientes } \\
\text { internados e as atitudes da equipe de } \\
\text { atendimento melhoraram }\end{array}$ \\
\hline $\begin{array}{c}\text { Improving Discharge } \\
\text { Efficiency in Medically } \\
\text { Complex Pediatric Patients } \\
\text { Statile, A. M. et al. (2016) } \\
\text { Estados Unidos }\end{array}$ & $\begin{array}{l}\text { Otimizar a porcentagem de pacientes } \\
\text { com medicamentos hospitalares } \\
\text { complexos que receberam alta em } 2 \\
\text { horas após o cumprimento das metas } \\
\text { de alta médica }\end{array}$ & $\begin{array}{l}\text { As metas de alta médica foram atingidas de } \\
50 \% \text { para } 80 \% \text { ao longo de } 17 \text { meses e se } \\
\text { mantiveram por mais } 6 \text { meses. Na } \\
\text { comparação pré-intervenção-pós- } \\
\text { intervenção, o tempo médio de permanência } \\
\text { não mudou e a taxa de readmissão de } 30 \text { dias } \\
\text { não foi afetada. }\end{array}$ \\
\hline
\end{tabular}

Fonte: Autores (2020).

\section{Discussão}

Partindo dos achados da pesquisa em questão, a discussão foi categorizada em tópicos envolvendo as práticas multiprofissionais: Equipe Multiprofissional; Relações interpessoais: equipe multiprofissional e família; Desafios e possibilidade das práticas multiprofissionais.

\section{Equipe Multiprofissional}

A sistematização do processo de trabalho da equipe multiprofissional decorre da compreensão dos profissionais envolvidos acerca do significado de trabalhar em equipe, bem como da sua relevância que reflete diretamente na qualidade de assistência permeando assim, uma atenção integral frente às necessidades demandadas pela criança/adolescente adoentada e pelo cuidador/familiar (Machado, Nóbrega, Silva, França, Reichert, \& Collet, 2018). 
Desse modo, os profissionais frequentemente apontam dificuldades que impactam na implementação de uma rotina para essa equipe multiprofissional. Das quais destacam-se as fragilidades de interação, na interdisciplinaridade e comunicação entre os integrantes da equipe; além da sobrecarga de trabalho que reverbera na redução de disponibilidade de tempo para possibilitar uma assistência mais eficaz ao cuidador/criança/adolescente. Outros aspectos que corroboram nesse processo é a burocratização dos serviços de saúde, fragilizando assim o desempenho do serviço e a qualidade da assistência (Machado, Nóbrega, Silva, França, Reichert, \& Collet, 2018).

Em consonância com este achado, Machado, Nóbrega, Silva, França, Reichert, e Collet (2018) afirmam que um serviço com todas essas problemáticas elencadas, apresenta falhas no acolhimento e no apoio social. Sendo assim, esses fatores podem potencializar o sofrimento da criança/adolescente e da sua família dificultando também no enfrentamento do adoecimento, como o consequente sentimento de tristeza e medo, a relação conflituosa entre profissionais e a família e o sentimento de subjugação dos serviços de saúde.

Diante deste contexto é sugestivo aos profissionais de saúde um modelo de atenção mais contínuo e efetivo, em que fica evidente uma transformação do modelo hegemônico das práticas de atenção à saúde centrada na doença e na execução de procedimentos, para um modelo entendido a partir das diretrizes do acolhimento, integralidade e responsabilização que passam pela formação de uma nova consciência no fazer e no saber profissional.

Em um estudo desenvolvido por Dalesio et al. (2019) um programa pediátrico de vias aéreas difíceis (PDART) foi criado, como uma forma de otimizar o manejo seguro de emergências de vias aéreas, sendo composto por uma equipe multidisciplinar que era treinada para ofertar esse atendimento conjunto, o que resultou em uma atuação mais eficaz e rápida, considerando o trabalho em conjunto e direcionado ao método abordado. Dessa forma, observou-se que a iniciativa de implementação de um serviço multidisciplinar e a educação em saúde ofertada aos componentes desse grupo, teve eficácia e seguridade do trabalho alcançando a integralidade da assistência.

As equipes multiprofissionais exitosas são deliberadas em seu trabalho conjunto, mesmo quando esse trabalho é limitado no tempo a um projeto específico. Eles identificam as funções da equipe, discutem os padrões de trabalho, criam uma compreensão de como as decisões serão feitas, sendo direcionadas por um representante, com atribuição de convocar as reuniões, facilitar a comunicação e direcionar a tomada de decisão do processo de trabalho; possibilitando um relacionamento longitudinal com o paciente. (Baird, Ashland, Rosenbluth, 2019).

\section{Relações interpessoais: equipe multiprofissional e família}

O processo de hospitalização da criança é uma associação de diversos fatores, tais quais: biológico, psicológico, social e ambiental. Desse modo, inserido a este processo temos a família da criança, que carrega a angustia e exaustão causados pelos fatores que compreendem a hospitalização interferindo diretamente no funcionamento familiar. (Broder, Crerand, Ruff, Peshansky, Sarwer \& Sischo, 2017; Vega et al., 2018).

Assim, a família tem necessidades específicas de cuidado que devem ser visíveis a todos os profissionais, independente do estágio da doença. É no procedimento de acolher com responsabilidade e resolutividade no hospital que o vínculo profissional-família é firmado de maneira válida e eficaz, proporcionando a diminuição de consequências geradas pela doença e auxiliando a família no reconhecimento dos profissionais como fonte de apoio (Swallow et al., 2014; Giraldo et al., 2015; Walter et al., 2019).

É ofertado pela equipe multiprofissional o apoio social, significando a parte elementar do processo de trabalho desenvolvido por esta equipe, desse modo, faz-se necessário a utilização da escuta qualificada, estabelecer vínculos entre equipe-família, consequentemente, otimizar o acesso aos serviços ofertados e assim, possibilitar um contexto favorável às 
experiências expressadas por meio da totalidade que compreende cada família. (Machado, Nóbrega, Silva, França, Reichert, \& Collet, 2018; Vaisbich et al., 2018).

Nessa perspectiva, a equipe multiprofissional procura imprimir dentro da sua proposta de trabalho ações que estimulem a inserção da família, que passa a desempenhar um papel de protagonista do cuidado da criança elevando os níveis de corresponsabilidade. A participação familiar é capaz de intensificar as reflexões acerca da compreensão de ações práticas sobre o cuidado e transformam as relações estabelecidas entre pacientes e profissionais. (Silva, Issi, Motta \& Botene, 2015).

A permanência em um ambiente hospitalar seja longa ou recorrente possibilita aos profissionais e familiares de crianças e adolescentes a oportunidade de fortalecer vínculos entre si gerando uma melhor adaptação frente a situação de hospitalização. Para haver uma responsabilização do cuidado é necessário que o profissional esteja disposto a construir um ambiente de confiança com família, nesse caso o diálogo marcado pela escuta qualificada torna-se um mecanismo significativo para favorecer o trabalho da equipe multiprofissional (Machado, Nóbrega, Silva, França, Reichert, \& Collet, 2018; Schneider, Pfister, Wörner, \& Ruppen, 2019).

Apenas o fortalecimento do vínculo estabelecido entre equipe e a família direcionará os meios necessários para proporcionar a oferta de apoio necessária, o qual evidência a importância de ações que centralizam a família, como também o cumprimento de intervenções parentais para propiciar o desenvolvimento de competências essenciais ao cuidado sendo um importante meio para redução do estresse familiar e ainda para melhora do bem-estar no ambiente hospitalar (Machado, Nóbrega, Silva, França, Reichert, \& Collet, 2018).

Desse modo, Balbino, Yamanaka, Balieiro e Mandetta (2015) apontam em seu estudo uma experiência com um filho recém-nascido (RN) na unidade neonatal. A família vivencia uma situação de muito sofrimento, porque se sente desesperada e insegura nesse ambiente. A busca por suporte de outros familiares e da equipe de saúde torna-se parte do enfrentamento dessa família.

Reduzir a ansiedade dos pais pelo oferecimento de apoio, esclarecimento de dúvidas, auxiliando-os a expressarem seus sentimentos, não só favorecem o vínculo afetivo entre pais e bebê, mas também para redução do estresse e, ainda, como preparar a família para o cuidado ao RN no domicílio, para isso ser atingido intervenções vêm sendo recomendadas e implantadas em unidades neonatais de algumas instituições, tais como: incentivo ao uso do método canguru, modificação de regras e rotinas restritivas que afastam a família por outras que a incluem como o incentivo a entrada para visitação de outros membros da família, a permanência dos pais com o filho internado em tempo integral, incentivo à participação dos pais nos cuidados ao bebê, nas tomadas de decisão do tratamento e implantação de espaços para ouvir a família em grupos de apoio (Balbino, Yamanaka, Balieiro \& Mandetta, 2015).

Com intuito de diminuir as barreiras que dificultam o estreitamento do vínculo entre equipe e familiares, Scotten, Manos, Malicoat, e Paolo (2015), enfatizam na utilização da telessaúde após a alta pediátrica da unidade de internação, comunicando equipe de internação com a família. As ligações de telessaúde são sempre interprofissionais, as chamadas incluem duas ou mais profissões e variam de uma a várias chamadas de acompanhamento, dependendo das necessidades do paciente e da família.

Segundo Baird, Ashland e Rosenbluth, (2019) a mudança de multidisciplinar para interprofissional está ocorrendo. A incorporação das famílias também está ganhando força, exigindo esforços ponderados e deliberados para nivelar hierarquias tradicionais e acomodar pontos de vista novos e diversos. Esses esforços irão produzir coletivamente um sistema de entrega de saúde que seja mais responsivo as necessidades dos pacientes e familiares e que oferece de forma mais consistente, seguro e de alta qualidade do cuidado.

Nos estudos encontrados, as reuniões familiares ganharam destaque como uma prática essencial para atender as demandas que emergem durante a hospitalização e até a alta. Conforme o estudo de Walter et al. (2019), a práticas de reuniões 
com familiares rotineiramente, são capazes de favorecer a melhoria do cuidado, uma vez que potencializam a compreensão do processo de adoecimento, possibilitando uma emancipação e o protagonismo e valorizando a corresponsabilidade diante do ato de cuidar, conforme Silva, Issi, Motta e Botene (2015).

Ainda nesse contexto, Walter et al. (2019) e Statile et al. (2016), destacam diante o desenvolvimento das reuniões, a contribuição para a tomada de decisão. As práticas desenvolvidas pela equipe multiprofissional, oportunizam em razão da comunicação direta entre equipe-família, uma percepção clara e coesa no que compete a diagnóstico, adesão ao tratamento e sobretudo as práticas que envolvem o cuidado além hospital.

Ressaltam-se os grupos de apoio, locais de esclarecimentos de dúvidas, onde começa a entender o que está ocorrendo. Com base nesse entendimento, a família passa a perceber a ajuda e a segurança para melhor enfrentamento do momento que está vivenciando, sentindo-se mais fortalecida por compartilhar suas experiências com os profissionais e com outras famílias. A proximidade entre família e profissionais da equipe presentes nas reuniões do grupo de apoio favorece o estreitamento das relações, o que torna a família mais segura e a faz atribuir ao grupo o significado de uma experiência transformadora (Balbino, Yamanaka, Balieiro \& Mandetta, 2015).

\section{Desafios e possibilidades das práticas multiprofissionais}

O estudo de Broder, Crerand, Ruff, Peshansky, Sarwer e Sischo (2017) descreveu alguns desafios previstos, imprevistos e estratégias para gerenciar estudos de longo prazo na população pediátrica com uma condição crônica de saúde: a fissura labial e/ou palatina. Embora a enfoque do estudo em questão seja o trato em uma população específica, as colocações e soluções descritas muito auxiliam o direcionamento para outras populações de pacientes crônicos pediátricos, atingindo o objetivo proposto.

Dentre os desafios previstos foram apresentados: estabelecimento de uma comunicação eficaz, adesão ao protocolo, garantir a qualidade dos dados, maximizar a retenção dos participantes e estudo da rotatividade de pessoal (Broder, Crerand, Ruff, Peshansky, Sarwer \& Sischo, 2017).

Estabelecer uma comunicação eficaz, proporcionou uma conclusão bem sucedida do projeto por meio do estabelecimento de uma comunicação regular, flexibilizada e que permitia um relacionamento sólido entre os membros da equipe do estudo e os participantes. A adesão ao protocolo utilizado, devido às diferenças do pessoal entre os locais, depende de uma organização prévia dos fatores que podem interferir nesse processo, como por exemplo, algumas atribuições podem ser conferidas por pessoas diferentes, dessa forma antes de iniciar o estudo, os integrantes multidisciplinares compartilharam apresentações entre a equipe, proporcionando revisar, calibrar os componentes da pesquisa e delegar as funções e deveres de cada indivíduo de forma coletiva (Broder, Crerand, Ruff, Peshansky, Sarwer \& Sischo, 2017).

Ainda se destacou nos artigos verificados a garantia da qualidade dos dados, de forma que os estatísticos revisaram os manuais de teste do instrumento visando garantir a validação e precisão da entrada de dados e seu gerenciamento. É de suma importância a maximização da retenção de participantes em acompanhamento de longo tempo, com utilização de boletins informativos sobre o estudo e aumento de vínculo valorizando a participação dos jovens no estudo. Por fim, a rotatividade de pessoal é um ponto que pode ser solucionado, segundo o estudo, com organização da equipe com materiais que permitam o treinamento de novos profissionais garantindo a continuidade do estudo (Broder, Crerand, Ruff, Peshansky, Sarwer \& Sischo, 2017).

\section{Conclusão}

Diante do estudo observa-se a importância da atuação multiprofissional na operacionalização e execução das atividades hospitalares, bem como destacam-se as relações interpessoais construídas mediante a aplicação das práticas e 
intervenções desenvolvidas nesse contexto, em favor da melhoria do processo de hospitalização, na compreensão do processo saúde-doença e todos os fatores a esses associados.

É necessário lidar com desafios para manter-se um cuidado efetivo e que proporcione uma prática mais organizada, com percepção aos fatores que necessitam ser revisados e modificados ao longo do processo de cuidado a fim de proporcionar um atendimento mais direcionado em que a equipe participe e se empenhe coletivamente.

No entanto, é oportuno salientar a necessidade de novos estudos que transcorram acerca da temática envolvendo a pediatria, apontando a importância da inserção de práticas multiprofissionais nesses serviços de saúde, para obtenção de uma assistência integral e eficaz à criança/adolescente hospitalizado.

\section{Referências}

Arnemann, C. T., Kruse, M. H. L., Gastaldo, D., Jorge, A. C. R., Silva, A. L., Margarites, A. G. F., Pires, C. L., Kuplich, N, M,, Santos, M, T, dos., \& Condessa, R, L. (2018). Práticas exitosas dos preceptores de uma residência multiprofissional: interface com a interprofissionalidade. Interface Comunicação, Saúde, Educação, 22(2), 1635-1646. https://dx.doi.org/10.1590/1807-57622017.0841.

Baird, J., Ashland, M., \& Rosenbluth, G. (2019). Interprofessional Teams: Current Trends and Future Directions. Pediatric clinics of North America, 66(4), 739-750. https://doi.org/10.1016/j.pcl.2019.03.003.

Balbino, F. S., Yamanaka, C. I., Balieiro, M. M. F. G., \& Mandetta, M. A. (2015). Grupo de apoio aos pais como uma experiência transformadora para a família em unidade neonatal. Escola Anna Nery, 19(2), 297-302. https://doi.org/10.5935/1414-8145.20150040.

Balsamo, L., Shabanova, V., Carbonella, J., Szondy, M. V., Kalbfeld, K., Thomas, D. A., Santucci, K., Grossman, M., \& Pashankar, F. (2019). Improving Care for Sickle Cell Pain Crisis Using a Multidisciplinary Approach. Pediatrics, 143(5), e20182218. https://doi.org/10.1542/peds.2018-2218.

Broder, H. L., Crerand, C. E., Ruff, R. R., Peshansky, A., Sarwer, D. B., \& Sischo, L. (2017). Challenges in conducting multicentre, multidisciplinary, longitudinal studies in children with chronic conditions. Community dentistry and oral epidemiology, 45(4), 317-322. https://doi.org/10.1111/cdoe.12293.

Carvalho, R. M. C., Cardoso, E. L. S, Ferreira, B. M. S, \& Ferreira, R. K. G. (2020). COVID-19 na pediatria: uma revisão integrativa. Research, Society and Development, 9 (9), e322997140. http://dx.doi.org/10.33448/rsd-v9i9.7140.

Dalesio, N. M., Diaz-Rodriguez, N., Koka, R., Kudchadkar, S., Mark, L. J., Cover, R., Pandian, V., Tunkel, D., \& Brown, R. (2019). Development of a Multidisciplinary Pediatric Airway Program: An Institutional Experience. Hospital pediatrics, 9(6), 468-475. https://doi.org/10.1542/hpeds.2018-0226

Dutra De Sousa, Lenice, Calcagno Gomes, G., Santos Da Silva, M. R., Pinto Dos Santos, C., \& Tarouco Da Silva, B. (2011). A família na unidade de pediatria: percepções da equipe de enfermagem acerca da dimensão cuidadora. Ciencia y enfermería, 17(2), 87-95. https://dx.doi.org/10.4067/S071795532011000200010 .

Giraldo Villa, A., Martínez Volkmar, M. I., Valencia Quintero, A. F., Montoya Delgado, D. C., Henao Roldan, C., Ruiz Navas, P., García Loboguerrero, F., \& Contreras Ramírez, M. M. (2015). Falla intestinal en el paciente pediátrico: experiencia y manejo por un grupo multidisciplinario [intestinal failure in pediatric patients: experience and management by a multidisciplinary group]. Nutricion hospitalaria, 32(6), 2650-2657. https://doi.org/10.3305/nh.2015.32.6.9725.

Lei 8080 de 19 de setembro de 1990, http://www.planalto.gov.br/ccivil_03/leis/18080.htm.

Liberati, A., Altman, D. G., Tetzlaff, J., Mulrow, C., Gøtzsche, P. C., Ioannidis, J. P., Clarke, M., Devereaux, P. J., Kleijnen, J., \& Moher, D. (2009). The PRISMA statement for reporting systematic reviews and meta-analyses of studies that evaluate health care interventions: explanation and elaboration. $P L O S$ Medicine, 6(7), e1000100.

Machado, A. N., Nóbrega, V. M., Silva, M. E. de A., França, D. B. L., de Reichert, A. P. S., \& Collet, N. (2018). Doença crônica infantojuvenil: vínculo profissional-família para a promoção do apoio social. Revista Gaúcha de Enfermagem, 39, e2017-0290. Epub August 02, 2018. https://dx.doi.org/10.1590/1983-1447.2018.2017-0290.

Peduzzi, M. (2007). Trabalho em equipe sob o eixo da integralidade: valores, saberes e práticas: ABRASCO.

Pereira, A. S., Shitsuka, D. M., Parreira, F. J. \& Sitsuka, R. (2018). Metodologia da pesquisa científica. UFSM. https://repositorio.ufsm.br/bitstream/handle/1/15824/Lic_Computacao_Metodologia-Pesquisa-Cientifica.pdf?sequence=1

Miareli, A. V. T. C. (2012) Trabalho multiprofissional na ESF: dificuldades ou desafios? Monografia (Especialização em Atenção Básica em Saúde da Família), Universidade Federal de Minas Gerais, Campos Gerais, Minas Gerais.

Schneider, T., Pfister, D., Wörner, A., \& Ruppen, W. (2019). Characteristics of children and adolescents at the Switzerland-wide first ambulatory interdisciplinary pain clinic at the University Children's Hospital Basel - a retrospective study. Swiss medical weekly, 149, w20073. https://doi.org/10.4414/smw.2019.20073.

Scotten, M., Manos, E. L., Malicoat, A., \& Paolo, A. M. (2015). Minding the gap: Interprofessional communication during inpatient and post discharge chasm care. Patient education and counseling, 98(7), 895-900. https://doi.org/10.1016/j.pec.2015.03.009.

Silva, A. F. da., Issi, H. B.r, Motta, M. da G. C. da., \& Botene, D. Z. de A.. (2015). Cuidados paliativos em oncologia pediátrica: percepções, saberes e práticas na perspectiva da equipe multiprofissional. Revista Gaúcha de Enfermagem, 36(2), 56-62. https://doi.org/10.1590/1983-1447.2015.02.46299. 
Research, Society and Development, v. 10, n. 3, e6810313052, 2021

(CC BY 4.0) | ISSN 2525-3409 | DOI: http://dx.doi.org/10.33448/rsd-v10i3.13052

Statile, A. M., Schondelmeyer, A. C., Thomson, J. E., Brower, L. H., Davis, B., Redel, J., Hausfeld, J., Tucker, K., White, D. L., \& White, C. M. (2016). Improving Discharge Efficiency in Medically Complex Pediatric Patients. Pediatrics, 138(2), e20153832. https://doi.org/10.1542/peds.2015-3832.

Swallow, V., Smith, T., Webb, N. J., Wirz, L., Qizalbash, L., Brennan, E., Birch, A., Sinha, M. D., Krischock, L., van der Voort, J., King, D., Lambert, H., Milford, D. V., Crowther, L., Saleem, M., Lunn, A., \& Williams, J. (2015). Distributed expertise: qualitative study of a British network of multidisciplinary teams supporting parents of children with chronic kidney disease. Child: care, health and development, 41(1), 67-75. https://doi.org/10.1111/cch.12141.

Vaisbich, M. H., Satiro, C. A. Fernandes, R. D., Nunes, D. de A. D., Messa, A. C. H. L., Lanetzki, C., \& Ferreira, J. C. de O. A.. (2019). Abordagem multidisciplinar para pacientes com cistinose nefropática: modelo para atendimento em uma doença renal rara e crônica. Brazilian Journal of Nephrology, 41(1), 131-141.https://dx.doi.org/10.1590/2175-8239-jbn-2018-0139.

Vega, E., Beaulieu, Y., Gauvin, R., Ferland, C., Stabile, S., Pitt, R., Gonzalez Cardenas, V. H., \& Ingelmo, P. M. (2018). Chronic non-cancer pain in children: we have a problem, but also solutions. Minerva anestesiologica, 84(9), 1081-1092. https://doi.org/10.23736/S0375-9393.18.12367-4.

Walter, J. K., Sachs, E., Schall, T. E., Dewitt, A. G., Miller, V. A., Arnold, R. M., \& Feudtner, C. (2019). Interprofessional Teamwork During Family Meetings in the Pediatric Cardiac Intensive Care Unit. Journal of pain and symptom management, 57(6), 1089-1098. https://doi.org/10.1016/j.jpainsymman.2019.03.002. 